\title{
Glutamate and Vivian Teichberg: a story about science, medicine, memory and love
}

\author{
Mia Levite Teichberg $\cdot$ Peter Riederer
}

Published online: 1 August 2014

(c) Springer-Verlag Wien 2014

\section{Glutamate}

Glutamate is a fascinating molecule, and an extremely important one, due to its numerous effects in the body. Glutamate has many different faces, some unveiled and pretty well known and understood, and some still mysterious and keep attracting numerous scientists, clinicians, pharmacologists and drug designers from all over the world for dozens of years.

Glutamate is the most important and abundant excitatory Neurotransmitter in the vertebrate nervous system. It plays a key role in long-term potentiation, in learning and memory and in many more neuronal functions. As such, Glutamate is involved in most aspects of the normal brain function, and in the development of the Central Nervous System (CNS).

But the function of Glutamate extends beyond the CNS, as it also plays a signaling role in peripheral organs and tissues among them the heart, kidney, intestine, lungs,

Editorial for the special issue on Glutamate in the Journal of Neural Transmission, in memory of Prof. Vivian Teichberg.

M. L. Teichberg ( $₫)$

School of Behavioral Sciences, Academic College of Tel-Aviv-

Yaffo, Tel Aviv, Israel

e-mail: mialevite@mta.ac.il

M. L. Teichberg

Faculty of Medicine, The Hebrew University of Jerusalem and

Hadassah Hebrew University Medical Center, Jerusalem, Israel

P. Riederer

Klinik und Poliklinik für Psychiatrie, Psychosomatik und

Psychotherapie des Universitätsklinikums Würzburg,

Füchsleinstrasse 15, 97080 Würzburg, Germany muscles, liver, ovary, testis, bone and pancreas and also in the adrenal, pituitary and pineal glands. Furthermore, Glutamate also induces direct and potent effects on most if not all cells of the immune system, as shown by a large body of evidence that accumulated in recent years. Due to these effects, we recently proposed to 'upgrade' Glutamate and recall it a 'NeuroImmunotransmitter' instead of a 'Neurotransmitter', since Glutamate seems to be very important for the ongoing function of the immune system, not only for that of the nervous system. This topic is discussed in the last part of this issue, which is devoted to Glutamate and its receptors in the immune system.

People often confuse Glutamate and Glutamic acid, so it may be useful to remind the readers of the relationship between the two, and here is a brief explanation: when the amino acid Glutamic acid which is acidic (and alike each amino acid, contains a central carbon atom, to which four different groups are bonded) loses a hydrogen from its side chain, it becomes Glutamate, with a side chain composed of $\mathrm{CH}_{2} \mathrm{CH}_{2} \mathrm{COO}^{-}$. In the human body, Glutamic acid almost always exists as Glutamate, because the conditions in the body favor the loss of the hydrogen atom from Glutamic acid, and it is Glutamate that is the Neurotransmitter that plays such a key role in the nervous system, in the immune system and in other body systems.

Interestingly, Glutamate is a striking example of how the mere concentration of a physiological molecule, and also the length of time it is 'seen' by target cells that are sensitive to it, dictate whether it induces beneficial and essential activity of the target cells and tissues, or rather very detrimental effects that finally sentences them to death. Thus, Glutamate in normal, physiological and regulated concentration induces beneficial effects that are absolutely essential for the ongoing function of the brain and of several peripheral organs. Yet, when Glutamate is 
in excess in the brain (occuring due to several different reasons in numerous pathological conditions), or if it is 'out there' for too long, it is catastrophic, since it induces overstimulation of Glutamate receptors leading to neurodegeneration and neuronal death through a process called 'Excitotoxicity'.

'Excitotoxicity', mediated by excess Glutamate and leading to a massive death of brain cells and loss of brain functions, plays a major pathological role in many human neurological diseases and injuries (as discussed in this issue for various neurological and psychiatric diseases).

For these reasons, lowering rapidly the excessive levels of Glutamate, or blocking Glutamate receptors to make them unresponsive to this excess, has been and remains a huge therapeutic challenge in so many neurological diseases and injuries/trauma.

Furthermore, Glutamate seems to play an important role in cancer, since it promotes tumor growth and/or spread of many types of tumor cells, while Glutamate antagonists block it (as discussed in one of the papers in this issue). Therefore, blocking Glutamate receptors, or lowering glutamate levels, seems to be an important therapeutic challenge in cancer too.

\section{The special Journal of Neuronal Transmission (JNT) issue on Glutamate}

This special issue on Glutamate, which was born thanks to the authors of this Editorial, deals with several very different aspects of Glutamate, and sheds light on some of glutamate's good and bad effects on different cells, tissues, systems and functions.

Scientists and clinicians from 10 countries (listed in alphabetical order): Finland, Germany, Hungary, Israel, Italy, Norway, Poland, Serbia and Montenegro, USA and United Kingdom, contributed to the 16 scientific papers of this issue. Yet, it should be kept in mind that Glutamate and its receptors could surely be discussed from many additional angles which are not discussed in this issue due to its space limitation. The same is true for the contribution of excess glutamate and 'Excitotoxicity' to many more neurological and psychiatric diseases which are unfortunately not covered in the present issue. Maybe, if this single issue leads to a series of additional issues on Glutamate, the newer ones will include papers on other interesting and important physiological and pathological aspects of Glutamate.

This issue on Glutamate is divided into few parts shedding light on different aspects of Glutamate.

The first part of this issue deals with Glutamate as a Neurotransmitter in the healthy brain, and discusses Glutamate receptors, effects and mechanism of action.

The second part deals with the pathological effects induced by excess Glutamate and 'Excitotoxicity', and with the primary role that 'Excitotoxicity' plays in many different human neurological and psychiatric diseases and in brain injury. Some of the papers included in this part of the issue also deal with a few therapeutic possibilities for blocking or silencing Glutamate receptors and Glutamate signaling when Glutamate is in excess, to minimize the resulting 'Excitotoxicity'.

The third part of the issue is devoted to the 'Brain to blood Glutamate scavenging' - the novel therapeutic approach developed by Prof. Vivian Teichberg, Israel (to whom this issue is dedicated, as discussed below) for preventing or minimizing the brain damage induced by excess Glutamate in many human diseases and injuries.

The fourth and last part of the issue deals with 'Glutamate and its receptors in the immune system', or in other words with 'Glutamate immunity and autoimmunity', and discusses this new field in science from three different angles: (a) Glutamate-induced beneficial effects on normal immune cells, which should be encouraged and could perhaps be used therapeutically for fighting cancer and infectious organisms (b) Glutamate-induced effects on autoimmune and cancerous immune cells, which are beneficial for these disease-causing immune cells (as they are for normal immune cells), but bad for health and as such should be arrested, (c) Autoimmune antibodies directed against Glutamate receptors in neurological and autoimmune diseases: the presence of various types of anti-Glutamate receptor antibodies in the serum and Cerebrospinal fluid (CSF) of patients with several types of neurological and autoimmune diseases, the multiple pathological effects that such antibodies induce in the brain (in animal models) and in tissue culture, their unique and exciting mechanism of action, and their removal or silencing (at least in some cases) by non specific immunotherapy. 


\section{Prof. Vivian Teichberg, to whom this special issue is dedicated}
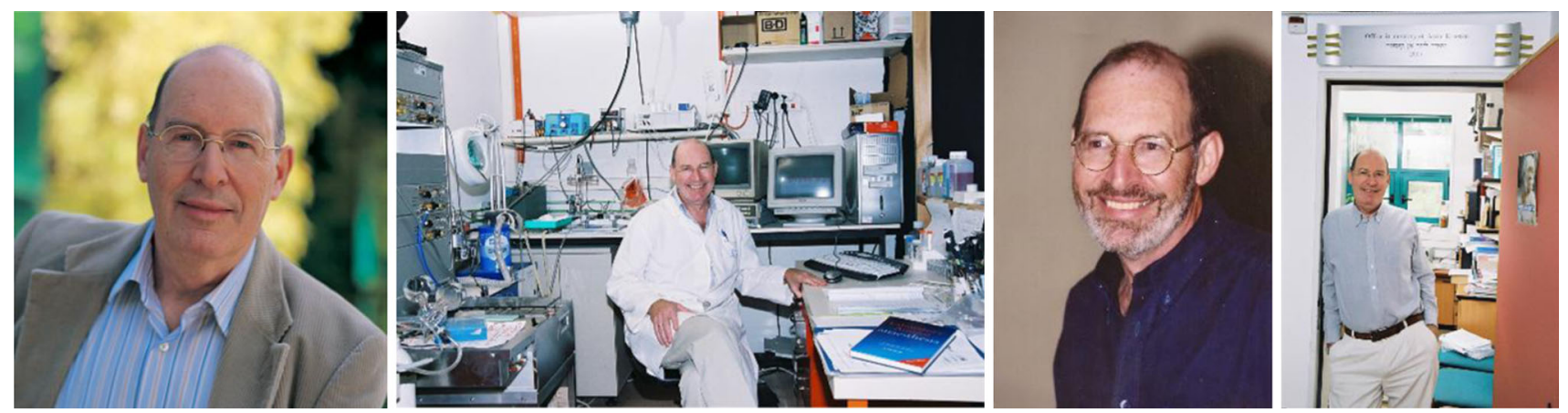

Vivian Teichberg, who held Belgian and Israeli nationalities, was born in Wattwill, Switzerland, in 1945, where his parents, having escaped from Belgium, took refuge from the Nazis. His mother Lili, a dentist, and his father, Yoel, a gynecologist, were both Holocaust survivors. The Teichberg family returned to Belgium after the second world war.

The Holocaust loomed large in Vivian's childhood and influenced his decision to move to Israel, as he believed that only there a Jew could truly be free. And indeed, Vivian moved from Belgium to Israel at the age of 21, and lived there till his death in 2011.

Vivian received an M.Sc. in Chemistry from the Free University of Brussels, Belgium in 1967. He continued his studies in Israel, and undertook his doctoral studies in Protein Chemistry and Biophysics at The Weizmann Institute of Science, Israel, earning a Ph.D. in 1972. In 1974, he followed to conduct Postdoctoral research in Neurochemistry at the Pasteur Institute in Paris for 3 years, in the laboratory of Prof. Jean Pierre Changeux who became Vivian's collaborator and personal friend for the rest of his life.

In 1977, after his Postdoctoral studies, Vivian returned to Israel, to the Weizmann Institute, and joined the Department of Neurobiology as a senior scientist. There, Vivian carried out pioneering studies, published many original papers, and based on all his achievements was promoted to Associate Professor in 1981, and to full Professor in 1992. He was also a visiting scientist at Stanford University, and at the Ecole Normale Supérieure in Paris.

In 1984, at the Weizmann Institute, Vivian met Mia Levite, who is a scientist-a Neuroimmunologist, and also a writer of poetry and prose books. Vivian and Mia married in 1985, and in later years their two lovely daughters were born: Ya'ar, born in 1987, who was an air traffic controller in her compulsory military service and now in her third year of studying industrial design, and Nof, born in 1995, who, following her father's footsteps, is now a paramedic in the navy of the Israeli army as part of her military service.
Vivian's interest in Neurochemistry and Neurobiology was originally triggered by the fact that his mother suffered from Parkinson's disease, and from his wish to find a cure for this debilitating illness. His research on the brain and on various Neurotransmitters and Neuropeptides led him to Glutamate and its receptors.

Vivian was among the first to isolate and study a receptor protein to which Glutamate binds in the brain, and to solve Glutamate receptor's primary structure-a seminal finding that contributed to the explosion of knowledge on the role of Glutamate receptors in brain physiology and pathology.

Vivian's scientific goal was to understand the basic structure of Glutamate receptors and the mechanism of action of Glutamate in the healthy brain. Furthermore, Vivian's therapeutic goal, which became his biggest applicable scientific and clinical mission, was to develop a novel methodology to prevent, or at least minimize, the catastrophic consequences in the brain caused by excess Glutamate and the ensuing 'Excitotoxicity' (which he nicknamed a 'Glutamate tsunami in the brain') in many neurological diseases and injuries.

Indeed, Vivian's strong ambition was to develop a treatment for various types of brain disorders among them Stroke, Traumatic brain injury, Amyotrophic Lateral Sclerosis (ALS), Lou Gehrig's disease and others, and also for brain tumors, among them brain glioma and others, all characterized by the presence of excess Glutamate in the brain fluids. Driven by this ambition, in the last $\sim 10$ years of his life, Vivian developed a novel and revolutionary therapeutic method which he called 'Brain to blood Glutamate scavenging' for removing the pathological excess Glutamate and preventing 'Excitotoxicity' in various different neurological diseases and injuries. This still experimental methodology is reviewed in the third part of this issue. Vivian based his therapeutic invention on the realization that first, the brain has natural 'pumps' that normally and physiologically 'suck up' Glutamate from the brain and transfer it to the blood when needed, and second, that when there is too much Glutamate in the brain, it may 
be possible to create therapeutic conditions, by introducing a putative drug, that will activate or speed up the function of the Glutamate pumps, and make them remove the excess Glutamate from the brain to the blood in an accelerated fashion, thus protecting the brain from 'Excitotoxicity'. Looking for novel means and putative drugs to speed up the activity of the Glutamate pumps and enhance the removal of excess glutamate from brain to blood, Vivian discovered he could inject into the blood a physiological enzyme called Glutamic Oxaloacetic Transaminase (GOT), which would then lower Glutamate levels in the blood, subsequently leading to a higher driving force for efflux (i.e. exit) of excess Glutamate from the brain to the blood.

In his pioneering paper on this topic, published in 2007 in Experimental Neurology, Vivian and his colleagues describe 'Brain neuroprotection by scavenging blood glutamate, and in another paper, published in 2011 in the Journal of Cerebral Blood Flow and Metabolism, which he entitled 'GOT to rid the body of excess glutamate' Vivian reviews this technology.

Over the years, in experiments performed in animal models, Vivian and his students and collaborators in Israel and abroad managed to prove the validity of this therapeutic 'Brain to blood Glutamate scavenging' method in animal models of few neuropathologies, and showed that it can lower Glutamate levels in the blood, which in turn lowers Glutamate levels in the brain, and subsequently confers brain protection in several brain pathologies among them brain trauma, Stroke, ALS and brain tumor (glioma).

During his life, Vivian made key discoveries, published numerous papers in top scientific journals, taught courses in Neuroscience, supervised in his lab many MSc and PhD students as well as Postdocs and clinicians, was a member of editorial boards of several international journals, and contributed to a large variety of national and international organizations and committees. Vivian was also the recipient of several highly regarded international prizes including the Somach Sachs Memorial Award and the French Academy of Sciences Professorship. At the Weizmann Institute, he was the incumbent of the Louis and Florence Katz-Cohen Professorial Chair of Neuropharmacology.
Vivian's last papers proved the validity of the 'Brain to blood glutamate scavenging' in animal models of several human diseases, and additional supporting studies he initiated or inspired continue to be published posthumously by his students, collaborators and others working on Glutamate and 'Excitotoxicity'.

Vivian died of metastatic melanoma in April 24th, 2011, when he was in one of the most exciting and rewarding peaks of his scientific career. During his illness, and despite many operations and other very harsh treatments, Vivian was still very active scientifically. He kept working on the 'Brain to blood Glutamate scavenging', as well as on several other topics.

Sadly, Vivian is not with us anymore, but his vision and therapeutic methodology to treat human pathologies caused by excess Glutamate live on. The Weizmann Institute of Science has carried on developing this methodology after Vivian's death and strives to bring this therapeutic approach to clinical trials. We hope that such clinical trials will indeed take place in the coming years, that they will be successful, that they will finally lead to the approval of the 'Brain to blood Glutamate scavenging' for use in patients with various neurological diseases and injuries, and that this novel therapeutic technology will save and improve the quality of lives of many people.

We also hope that this special JNT issue on Glutamate will be the first step towards the organization of an international interdisciplinary meeting on Glutamate in Vivian's memory. Such a meeting could enrich the scientific and medical community, a fitting tribute to a gifted and innovative scientist who made a valuable contribution to science, and who was also a wonderful, good-hearted, kind and modest human being loved deeply by his family, friends, students and collaborators.

May his soul rest in peace. Amen.

Mia Levite Teichberg, Tel Aviv, Israel

Peter Riederer, Würzburg, Germany

May, 2014 